\title{
Performance of North American Strawberry Cultivars under Conditions Mimicking California Production Systems
}

\author{
John M. Rariden and Douglas V. Shaw \\ Pomology Department, University of California, Davis, CA 95616 \\ Additional index words. adaptation, plant breeding, germplasm, genotype $\times$ location interaction
}

\begin{abstract}
Runner plants from 16 strawberry (Fragaria $\times$ ananassa Duch.) cultivars were grown using annual Mediterranean production systems to test for differences in productivity, performance traits, and vegetative growth attributes. Genotypes were included from germplasm adapted to four geographic regions: California and northwestern, northeastern, and mid-Atlantic or southeastern United States. The California genotypes were divided further into day-neutral and June-bearing categories. With these treatments, California cultivars had significantly larger plants and grew more rapidly during the fall and winter, had larger fruit, and produced at least twice the quantity of fruit of cultivars from the other regions. Variance components due to region explained $64 \%$ and $26 \%$ of the phenotypic variance for early and total yield, respectively, whereas differences among cultivars within regions explained $12 \%$ and $7 \%$ of the variance for these traits. Cultivars from all regions had significantly larger plants and were more productive when treated with 3 weeks of artificial vernalization. However, region $\times$ vernalization effects were nonsignificant for all traits, a result suggesting that selection in Mediterranean environments has not adapted germplasm specifically for low vernalization conditions.
\end{abstract}

Although considerable evidence exists for regional adaptations of physiological traits in North American strawberry cultivars (Arney, 1956; Darrow and Waldo, 1934; Guttridge, 1953; Kronenberg and Wassenaar, 1972), information about production traits is usually available only for cultivars tested in a rather narrow range of environments. The extent to which cultivars are physiologically adapted has ramifications for production efficiency, but direct comparisons of economically important characters may reveal more about the potential benefits of, and obstacles to, germplasm exchange.

Hancock et al. (1992) compared cultivars originally selected and tested under standard eastern (perennial) production systems with California cultivars selected for annual Mediterranean culture. Cultivars from both sources were grown with methods that simulated standard eastern production systems. Their results demonstrated little if any difference for yield between California and eastern cultivars, and they concluded that California types were not innately higher yielding than eastern cultivars. They interpreted this observation as evidence that the large differences in regional productivity-California yields of $>55 \mathrm{vs}$. $<20 \mathrm{t} \cdot \mathrm{ha}^{-1}$ for North America, excluding California (Hancock and Scott, 1989; Voth and Bringhurst, 1990)_are largely the consequences of differing environments. Because the genetic factors that determine strawberry plant growth and production capacity may interact substantially with such different environments, this question remains only partially resolved.

Northeastern and Mediterranean production environments are quite distinct, both physically and culturally, and the factors that condition adaptation to these differing environments are probably complex. Low-temperature tolerance and disease resistance are frequently cited as limiting adaptational features for eastern perennial culture (Galletta et al., 1989; Hancock et al., 1992), both of which were controlled in the comparisons made by Hancock et al. (1992). Conversely, the capability to sustain growth at low tem-

Received for publication 1 Dec. 1993. Accepted for publication 11 Feb. 1994. The cost of publishing this paper was defrayed in part by the payment of page charges. Under postal regulations, this paper therefore must be hereby marked advertisement solely to indicate this fact. peratures and the capacity to perform with minimal vernalization have been suggested as essential characteristics of cultivars adapted to Mediterranean production systems (Bringhurst and Voth, 1984; Galletta and Bringhurst, 1990).

In fact, variation for strawberry plant growth characteristics and resource partitioning patterns has been found within and among germplasm sources, and, in several cases, innate growth differences have been related to differences in productivity (Shaw, 1993; Strik and Proctor, 1988b). Hancock et al. (1992) found that California cultivars allocated a significantly higher fraction of their growth potential to reproductive effort than eastern cultivars. Also, although the results were not uniformly significant, eastern cultivars typically had larger plant dry weights, more crowns per plant, and more total leaf area. Because Strik and Proctor (1988a) and Shaw (1993) demonstrated that vegetative growth during critical periods can condition a substantial fraction of the observed variation for seasonal yield, it is likely that adaptational features may primarily reflect vegetative growth patterns.

The purpose of the experiments described below was to provide complementary information to the results cited above (Hancock et al., 1992) by testing cultivars from several North American sources in annual Mediterranean production systems that included two vernalization treatments. Although the primary focus of this research was to evaluate production characteristics, differences among sources for vegetative traits were examined as well.

\section{Materials and Methods}

Plant material and cultural treatments. Identities and categorical descriptions of the 16 cultivars included in our experiment are given in Table 1. Cultivars were divided into five subgroups primarily based on their geographic region of origin or principal region of cultivation (Chandler, 1991; Sjulin and Dale, 1987). Because differences in vegetative growth characteristics have been reported among cultivars of differing photoperiodic types (Nicoll and Galletta, 1987), California cultivars were classified further as either June-bearing or day-neutrals. All non-Californian cultivars were June-bearing.

Forty (occasionally fewer) runner plants for each cultivar were 
Table 1. Cultivars and selections classified by geographic region and photoperiodic type.

\begin{tabular}{lcc}
\hline \hline $\begin{array}{l}\text { Geographic } \\
\text { region }\end{array}$ & $\begin{array}{c}\text { Photoperiodic } \\
\text { type }\end{array}$ & Cultivars \\
\hline California & June-bearing & Tioga, Pajaro, Douglas, Chandler \\
California & Day-neutral & Selva, Irvine, Seascape, Capitola \\
Pacific northwest & Benton, Hood, Totem \\
Northern-northeastern & & Earliglow, Honeoye, Kent \\
Southeast-lower midwestern-mid-Atlantic & Allstar, Guardian \\
\hline
\end{tabular}

harvested from the Univ. of California, Davis (UCD), Pomology Dept.'s Wolfskill Experimental Orchard in Winters, Calif., on 24 Oct. 1991. On this date, plants at this location had received no natural vernalization (exposure to $<7.2 \mathrm{C}$ ). All plants were pruned at harvest to retain two leaves and were established using two-row diagonal beds on $1.5-\mathrm{m}$ centers, with $33 \mathrm{~cm}$ between plants along rows and $30 \mathrm{~cm}$ between rows (Welch, 1989). Half of the plants for each cultivar received minimal cold storage ( $2 \mathrm{C}$ for $\approx 3$ days) and were planted on 28 Oct. 1991 at the UCD Strawberry Research Facility in Watsonville, Calif. The remaining plants were stored at 2C and planted on 13 Nov. after 3 weeks ( $500 \mathrm{~h})$ of cold storage. A single plot of 10 plants (occasionally fewer) for each cultivar was established in each of two blocks per planting date. Clear polyethylene bed mulch was applied to the beds within 2 weeks of planting to stimulate plant growth through the winter (Voth and Bringhurst, 1990).

Data for growth and productivity traits were collected during the annual season. Vegetative growth measurements were obtained for individual plants on $15 \mathrm{Feb}$. to describe plant growth from planting to winter rest (fall and winter diameter increment) and on 8 Apr. to quantify vegetative activity further through the early flowering and production season (Shaw, 1993). The last measurement date represents the final or stable plant size, at which time plants filled their allocated space. Although plants in Mediterranean environments continue to increase in size after early spring and this growth may be important to late-season yield (Shaw, 1993), additional measurements were judged unreliable as indicators of vegetative activity because of interplant competition. Plant cross-sectional diameters were measured on both dates and the crowns were counted at the final measurement (Shaw, 1993). Diameters obtained on 15 Feb. were reduced to plot means for further analyses. Changes in plant size between 15 Feb. and 8 Apr. were first transformed to relative diameter increases (Roberts et al., 1985) to avoid confounding with treatments that affected establishment plant growth, then expressed as plot means. Plot yield, fruit size (10 berry weight), and a commercial appearance score ( 1 to 5 scale) were obtained for 20 consecutive weeks beginning 7 Apr. In addition, fruit firmness was obtained for a single representative fruit from each plot on 10 of the 20 harvest dates using a UC penetrometer. Production traits were generated and evaluated as early (first 6 weeks) and seasonal (total) yield.
Fruit size and appearance scores were expressed as weighted averages, with weighting performed using weekly yield values.

Experimental design and statistical analysis. Analyses of variance (ANOVAs) were conducted on the combined data set with block and vernalization treatment (planting date and length of cold storage) treated as fixed effects. Geographical regions, genotypes nested in regions, and their interactions with vernalization treatment were included as random effects. Statistical tests were conducted and model variance components were calculated using linear functions of the expected mean squares given in Table 2 (Searle, 1971); components of variance for individual sources $\left(\sigma_{i}^{2}\right)$ were expressed as a percentage of the phenotypic variance of random effects:

$$
\sigma^{2}{ }_{\mathrm{i}} \%=\frac{\sigma_{\mathrm{i}}^{2}}{\sigma^{2}+\sigma_{\mathrm{C} / \mathrm{R}}^{2}+\sigma_{\mathrm{V} \times \mathrm{R}}^{2}+\sigma_{\mathrm{V} \times \mathrm{C} / \mathrm{R}}^{2}+\sigma^{2}}
$$

where $\sigma_{\mathrm{R}}^{2}, \sigma_{\mathrm{C} / \mathrm{R}}^{2}, \sigma_{\mathrm{V} \times \mathrm{R}}^{2}, \sigma_{\mathrm{V} \times \mathrm{C} / \mathrm{R}}^{2}$, and $\sigma^{2}$ are the components of variance for geographical region, cultivar in region, region $\times$ vernalization interaction, cultivar in subgroup $\times$ vernalization interaction, and experimental error, respectively.

Although the primary intent of our study was to evaluate genetic sources of variation combined with different vernalization treatments, it should be noted that vernalization treatments in this study were confounded with planting date. In addition to differential chilling, the length of the growth period after planting and the climatic conditions at establishment differed among treatments. This option allowed comparison of runner plants harvested at similar maturity and was judged the treatment closest to commercial practices (Voth and Bringhurst, 1990).

\section{Results and Discussion}

Means and standard deviations are given for production and vegetative traits by region and vernalization treatment in Table 3, and the response to vernalization treatment is summarized in Table 4. Growth during the fall and winter was greater for plants from all regions with longer vernalization periods. Likewise, there were more crowns in spring for all regional sources with greater vernalization. Apparently, plant vigor resulting from the longer vernalization period more than compensated for the 3-week shorter

Table 2. Expected mean squares for analysis of variance for seven vegetative growth and performance traits.

\begin{tabular}{lrl}
\hline \hline Source & df & \multicolumn{1}{c}{ Expected mean squares $^{\mathrm{z}}$} \\
\hline Block & 1 & $\sigma^{2}+\mathrm{Q}_{\mathrm{B}} \sigma^{2} \sigma^{2}+\mathrm{k}_{1} \sigma_{\mathrm{V} \times \mathrm{C} / \mathrm{R}}+\mathrm{k}_{3} \sigma_{\mathrm{V} \times \mathrm{R}}^{2}+\mathrm{Q}_{\mathrm{V}}$ \\
Vernalization (V) & 1 & $\sigma^{2}+\mathrm{k}_{1} \sigma_{\mathrm{V} \times \mathrm{C} / \mathrm{R}}+\mathrm{k}_{2} \sigma_{\mathrm{C} / \mathrm{R}}^{2}+\mathrm{k}_{3} \sigma_{\mathrm{V} \times \mathrm{R}}^{2}+\mathrm{k}_{4} \sigma_{\mathrm{R}}^{2}$ \\
Region (R) & 4 & $\sigma^{2}+\mathrm{k}_{1} \sigma_{\mathrm{V} \times \mathrm{C} / \mathrm{R}}^{2}+\mathrm{k}_{3} \sigma_{\mathrm{V} \times \mathrm{R}}^{2}$ \\
$\mathrm{~V} \times \mathrm{R}$ & 4 & $\sigma^{2}+\mathrm{k}_{1} \sigma_{\mathrm{V} \times \mathrm{C} / \mathrm{R}}+\mathrm{k}_{2} \sigma_{\mathrm{C} / \mathrm{R}}^{2}$ \\
Cultivar (C)/R & 10 & $\sigma^{2}+\mathrm{k}_{1} \sigma_{\mathrm{V} \times \mathrm{C} / \mathrm{R}}^{2}$ \\
$\mathrm{~V} \times \mathrm{C} / \mathrm{R}$ & 10 & $\sigma^{2}$ \\
Error & 59 &
\end{tabular}

${ }^{\mathrm{z} C o e f f i c i e n t s:} \mathrm{k}_{1}=2, \mathrm{k}_{2}=5.9, \mathrm{k}_{3}=4$, and $\mathrm{k}_{4}=11.9$. 
Table 3. Means and standard deviations (in parentheses) for production and vegetative growth of cultivars categorized by geographic region or photoperiodic type grown in the field with partial or no chilling.

\begin{tabular}{|c|c|c|c|c|c|c|c|c|c|}
\hline $\begin{array}{l}\text { Geographic } \\
\text { region or } \\
\text { photoperiod } \\
\text { type }\end{array}$ & $\begin{array}{l}\text { Days of } \\
\text { cold } \\
\text { storage }\end{array}$ & $\begin{array}{l}\text { Early } \\
\text { yield }^{z} \\
(\mathrm{~g})\end{array}$ & $\begin{array}{l}\text { Total } \\
\text { yield } \\
(\mathrm{g})\end{array}$ & $\begin{array}{l}\text { Fruit } \\
\text { size } \\
\text { (g/fruit) }\end{array}$ & $\begin{array}{l}\text { Appearance } \\
\text { score }\end{array}$ & Firmness & $\begin{array}{c}\text { Fall-winter } \\
\text { diam } \\
(\mathrm{cm})\end{array}$ & $\begin{array}{c}\text { Spring } \\
\text { relative } \\
\text { growth }(\%)\end{array}$ & $\begin{array}{c}\text { Spring } \\
\text { crown } \\
\text { no. }\end{array}$ \\
\hline \multirow[t]{2}{*}{ California June-bearing } & 0 & $\begin{array}{l}162.3 \\
(48.5)\end{array}$ & $\begin{array}{l}1108.5 \\
(381.3)\end{array}$ & $\begin{array}{l}22.18 \\
(1.73)\end{array}$ & $\begin{array}{c}3.13 \\
(0.24)\end{array}$ & $\begin{array}{c}5.97 \\
(1.18)\end{array}$ & $\begin{array}{l}296.8 \\
(49.1)\end{array}$ & $\begin{array}{c}3.27 \\
(1.02)\end{array}$ & $\begin{array}{c}3.17 \\
(1.02)\end{array}$ \\
\hline & 21 & $\begin{array}{l}150.1 \\
(26.0)\end{array}$ & $\begin{array}{l}1461.7 \\
(306.1)\end{array}$ & $\begin{array}{l}23.48 \\
(0.92)\end{array}$ & $\begin{array}{c}3.13 \\
(0.17)\end{array}$ & $\begin{array}{c}5.54 \\
(0.81)\end{array}$ & $\begin{array}{l}352.3 \\
(42.5)\end{array}$ & $\begin{array}{l}3.85 \\
(1.16)\end{array}$ & $\begin{array}{r}4.58 \\
(0.89)\end{array}$ \\
\hline \multirow[t]{2}{*}{ California day-neutral } & 0 & $\begin{array}{l}156.6 \\
(42.1)\end{array}$ & $\begin{array}{l}1375.2 \\
(449.2)\end{array}$ & $\begin{array}{l}21.21 \\
(2.08)\end{array}$ & $\begin{array}{c}3.11 \\
(0.16)\end{array}$ & $\begin{array}{c}6.64 \\
(1.41)\end{array}$ & $\begin{array}{l}275.8 \\
(28.9)\end{array}$ & $\begin{array}{c}3.36 \\
(1.50)\end{array}$ & $\begin{array}{c}3.31 \\
(0.72)\end{array}$ \\
\hline & 21 & $\begin{array}{l}191.7 \\
(83.9)\end{array}$ & $\begin{array}{l}1975.9 \\
(556.7)\end{array}$ & $\begin{array}{l}23.03 \\
(1.88)\end{array}$ & $\begin{array}{c}3.26 \\
(0.24)\end{array}$ & $\begin{array}{c}6.29 \\
(1.07)\end{array}$ & $\begin{array}{l}319.0 \\
(24.7)\end{array}$ & $\begin{array}{c}3.74 \\
(1.30)\end{array}$ & $\begin{array}{c}3.96 \\
(0.75)\end{array}$ \\
\hline \multirow[t]{2}{*}{ Pacific northwest } & 0 & $\begin{array}{c}30.0 \\
(14.0)\end{array}$ & $\begin{array}{c}267.9 \\
(182.2)\end{array}$ & $\begin{array}{l}12.77 \\
(1.11)\end{array}$ & $\begin{array}{c}2.55 \\
(0.16)\end{array}$ & $\begin{array}{c}4.82 \\
(0.88)\end{array}$ & $\begin{array}{l}220.8 \\
(25.1)\end{array}$ & $\begin{array}{c}6.08 \\
(1.59)\end{array}$ & $\begin{array}{c}3.11 \\
(1.02)\end{array}$ \\
\hline & 24 & $\begin{array}{c}54.0 \\
(26.4)\end{array}$ & $\begin{array}{c}383.6 \\
(235.6)\end{array}$ & $\begin{array}{l}14.72 \\
(1.24)\end{array}$ & $\begin{array}{c}2.35 \\
(0.17)\end{array}$ & $\begin{array}{c}4.79 \\
(0.65)\end{array}$ & $\begin{array}{c}298.5 \\
(9.0)\end{array}$ & $\begin{array}{c}5.23 \\
(1.32)\end{array}$ & $\begin{array}{c}4.73 \\
(0.98)\end{array}$ \\
\hline \multirow[t]{2}{*}{ Northern-northeastern } & 0 & $\begin{array}{c}37.1 \\
(40.4)\end{array}$ & $\begin{array}{c}377.3 \\
(148.6)\end{array}$ & $\begin{array}{l}14.40 \\
(1.64)\end{array}$ & $\begin{array}{c}2.55 \\
(0.42)\end{array}$ & $\begin{array}{c}5.57 \\
(0.93)\end{array}$ & $\begin{array}{l}196.7 \\
(34.2)\end{array}$ & $\begin{array}{c}6.27 \\
(3.12)\end{array}$ & $\begin{array}{c}2.45 \\
(0.64)\end{array}$ \\
\hline & 21 & $\begin{array}{c}79.1 \\
(35.2)\end{array}$ & $\begin{array}{c}554.2 \\
(189.9)\end{array}$ & $\begin{array}{l}16.84 \\
(1.30)\end{array}$ & $\begin{array}{c}2.66 \\
(0.29)\end{array}$ & $\begin{array}{c}5.59 \\
(0.75)\end{array}$ & $\begin{array}{l}269.6 \\
(33.8)\end{array}$ & $\begin{array}{c}4.52 \\
(1.00)\end{array}$ & $\begin{array}{c}3.19 \\
(0.87)\end{array}$ \\
\hline \multirow[t]{2}{*}{ Southeastern-mid-Atlantic } & 0 & $\begin{array}{l}26.6 \\
(20.2)\end{array}$ & $\begin{array}{c}379.3 \\
(190.6)\end{array}$ & $\begin{array}{l}15.02 \\
(2.17)\end{array}$ & $\begin{array}{c}2.40 \\
(0.12)\end{array}$ & $\begin{array}{c}6.78 \\
(0.58)\end{array}$ & $\begin{array}{l}193.3 \\
(18.7)\end{array}$ & $\begin{array}{c}3.52 \\
(0.10)\end{array}$ & $\begin{array}{c}2.21 \\
(0.35)\end{array}$ \\
\hline & 21 & $\begin{array}{c}58.4 \\
(54.1)\end{array}$ & $\begin{array}{c}718.9 \\
(141.0)\end{array}$ & $\begin{array}{l}19.60 \\
(2.43)\end{array}$ & $\begin{array}{c}2.35 \\
(0.10)\end{array}$ & $\begin{array}{c}6.58 \\
(1.25)\end{array}$ & $\begin{array}{l}312.5 \\
(51.3)\end{array}$ & $\begin{array}{c}3.37 \\
(0.39)\end{array}$ & $\begin{array}{c}3.59 \\
(1.06)\end{array}$ \\
\hline
\end{tabular}

${ }^{\mathrm{z} E a r l y}$ yield is that accumulated during the first 6 weeks of harvest.

growing season available to plants given longer storage periods. Relative spring diameter increments were larger for California cultivars with greater vernalization, but the converse was true for cultivars from other regions. Total yields and fruit size were larger for cultivars from all regions with greater vernalization, and early yields were enhanced by vernalization for all categories except California June bearers. Appearance scores and fruit firmness were largely unaffected by vernalization treatment.

Large differences were detected between regional categories for nearly all vegetative and production traits. California cultivars grew somewhat more during the fall and winter than those from other regions and had more crowns than both eastern sources (Table 5). This trend was consistent regardless of vernalization treatment (Table 3). Early and total yields for the California sources were at least twice that for any other region, and this pattern was also consistent, regardless of vernalization treatment (Table $3)$. Smaller absolute differences between California sources and the others were apparent for fruit size, and no trends were obvious for fruit firmness or spring diameter increment (Table 5).

ANOVA results confirmed that the main effects of vernalization were significant $(P<0.05)$ or highly significant $(P<0.01)$ for fall and winter growth, crown number, total yield, and weighted fruit size (Table 6). Despite large apparent mean differences for early yield, vernalization treatment was only borderline significant $(P<0.07)$, and no significant main effects of vernalization were detected for spring relative growth and fruit firmness.

The large differences among regional categories for fall and winter diameter increment, early and total yield, fruit size, and appearance were reflected in significant or highly significant variances among regions in ANOVAs (Table 6). Importantly, interactions between regional category and vernalization treatment were uniformly nonsignificant for growth and yield variables, a result suggesting the absence of innate differences among germplasm sources for response to partial vernalization. Signifi- cant variation was detected among cultivars within regional categories for all three vegetative traits, total yield, appearance score, and fruit firmness; several of the cultivar $\times$ vernalization interaction variances were significant as well (Table 6). The observation that cultivars from a single region interact with vernalization treatment, together with the absence of region $\times$ vernalization interactions, suggests that selection has done little to adapt California cultivars specifically to low chilling regimes.

Examining the variance components further resolves the trends apparent in mean comparisons and ANOVAs. A relatively small fraction of the phenotypic variance for vegetative traits was explained by among-region differences (5\% to 12\%) (Table 7), and a somewhat larger fraction was explained by differences among cultivars within regions (5\% to 20\%) (Table 7). Conversely, differences among regions explained $64 \%$ and $26 \%$ of the phenotypic variance for early and total yield, respectively, whereas differences among cultivars within regions explained $12 \%$ and $7 \%$ of the variance, respectively, for these traits. Large regional differences exist for production traits, but regional variation for vegetative growth characteristics is less important. Although California cultivars differ from those selected in other regions for vegetative and production traits, direct selection for productivity has had the largest impact.

This study evaluated the performance of 14 of the 20 mostcommonly planted public-sector cultivars (Chandler, 1991) and, although this represents a rather small sample of the available germplasm, the results revealed some clear trends. Whereas Hancock et al. (1992) found little evidence for regional differentiation of North American cultivars based on their performance in northeastern environments, our results demonstrate a clear advantage for California cultivars when evaluated in Mediterranean annual production environments. This discrepancy may be best explained as a classic scale interaction between eastern and Mediterranean environments: cultivars selected in northern temperate 
Table 4. Means and standard deviations (in parentheses) for production and vegetative growth of cultivars grown in the field with partial or no chilling.

\begin{tabular}{|c|c|c|c|c|c|c|c|c|}
\hline $\begin{array}{l}\text { Days of } \\
\text { cold } \\
\text { storage }\end{array}$ & $\begin{array}{c}\text { Early } \\
\text { yield }^{z} \\
(\mathrm{~g})\end{array}$ & $\begin{array}{c}\text { Total } \\
\text { yield } \\
(\mathrm{g})\end{array}$ & $\begin{array}{l}\text { Fruit } \\
\text { size } \\
\text { (g/fruit) }\end{array}$ & $\begin{array}{l}\text { Appearance } \\
\text { score }\end{array}$ & $\begin{array}{c}\text { Fruit } \\
\text { firmness }\end{array}$ & $\begin{array}{c}\text { Fall-winter } \\
\text { diam } \\
(\mathrm{cm})\end{array}$ & $\begin{array}{c}\text { Spring } \\
\text { relative } \\
\text { growth }(\%)\end{array}$ & $\begin{array}{c}\text { Spring } \\
\text { crown } \\
\text { no. }\end{array}$ \\
\hline \multirow[t]{2}{*}{0} & 91.2 & 768.0 & 17.53 & 2.79 & 5.95 & 242.2 & 4.49 & 2.92 \\
\hline & (73.3) & $(563.0)$ & $(4.30)$ & $(0.39)$ & $(1.25)$ & $(52.4)$ & $(2.19)$ & $(0.86)$ \\
\hline \multirow[t]{2}{*}{21} & 115.5 & 1102.7 & 19.76 & 2.81 & 5.74 & 310.8 & 4.16 & 4.03 \\
\hline & (76.2) & (731.7) & (3.87) & $(0.44)$ & (1.05) & $(41.2)$ & (1.24) & (1.01) \\
\hline
\end{tabular}

${ }^{\mathrm{z}}$ Early yield is that accumulated during the first 6 weeks of harvest.

Table 5. Means and standard deviations (in parentheses) for production and vegetative growth of cultivars categorized by geographic region or photoperiodic type grown in the field.

\begin{tabular}{|c|c|c|c|c|c|c|c|c|}
\hline $\begin{array}{l}\text { Geographic } \\
\text { region or } \\
\text { photoperiod } \\
\text { type }\end{array}$ & $\begin{array}{l}\text { Early } \\
\text { yield }^{\mathrm{z}} \\
(\mathrm{g})\end{array}$ & $\begin{array}{l}\text { Total } \\
\text { yield } \\
(\mathrm{g})\end{array}$ & $\begin{array}{c}\text { Fruit } \\
\text { size } \\
\text { (g/fruit) }\end{array}$ & $\begin{array}{c}\text { Appearance } \\
\text { score }\end{array}$ & $\begin{array}{c}\text { Fruit } \\
\text { firmness }\end{array}$ & $\begin{array}{c}\text { Fall-winter } \\
\text { diam } \\
(\mathrm{cm})\end{array}$ & $\begin{array}{c}\text { Spring } \\
\text { relative } \\
\text { growth }(\%)\end{array}$ & $\begin{array}{c}\text { Spring } \\
\text { crown } \\
\text { no. }\end{array}$ \\
\hline California June-bearing & $\begin{array}{l}156.2 \\
(37.6)\end{array}$ & $\begin{array}{l}1285.1 \\
(377.7)\end{array}$ & $\begin{array}{l}22.83 \\
(1.48)\end{array}$ & $\begin{array}{c}3.13 \\
(0.20)\end{array}$ & $\begin{array}{c}5.76 \\
(0.99)\end{array}$ & $\begin{array}{l}324.5 \\
(52.5)\end{array}$ & $\begin{array}{c}3.56 \\
(1.08)\end{array}$ & $\begin{array}{c}3.88 \\
(1.18)\end{array}$ \\
\hline California day-neutral & $\begin{array}{c}103774.2 \\
(66.7)\end{array}$ & $\begin{array}{c}1037,675.6 \\
(578.8)\end{array}$ & $\begin{array}{l}22.12 \\
(2.13)\end{array}$ & $\begin{array}{c}3.18 \\
(0.21)\end{array}$ & $\begin{array}{c}6.47 \\
(1.22)\end{array}$ & $\begin{array}{l}297.4 \\
(34.3)\end{array}$ & $\begin{array}{c}3.55 \\
(1.37)\end{array}$ & $\begin{array}{c}3.64 \\
(0.79)\end{array}$ \\
\hline Pacific northwest & $\begin{array}{c}42.0 \\
(23.7)\end{array}$ & $\begin{array}{c}325.7 \\
(209.7)\end{array}$ & $\begin{array}{c}13.74 \\
(1.51)\end{array}$ & $\begin{array}{c}2.45 \\
(0.19)\end{array}$ & $\begin{array}{c}4.80 \\
(0.74)\end{array}$ & $\begin{array}{l}269.7 \\
(44.4)\end{array}$ & $\begin{array}{c}5.66 \\
(1.46)\end{array}$ & $\begin{array}{c}3.92 \\
(1.27)\end{array}$ \\
\hline Northern-northeastern & $\begin{array}{c}58.1 \\
(42.3)\end{array}$ & $\begin{array}{c}465.8 \\
(187.0)\end{array}$ & $\begin{array}{c}15.62 \\
(1.90)\end{array}$ & $\begin{array}{c}2.60 \\
(0.35)\end{array}$ & $\begin{array}{c}5.58 \\
(0.81)\end{array}$ & $\begin{array}{l}233.2 \\
(50.0)\end{array}$ & $\begin{array}{c}5.39 \\
(2.39)\end{array}$ & $\begin{array}{c}2.82 \\
(0.83)\end{array}$ \\
\hline Southeastern-mid-Atlantic & $\begin{array}{c}42.5 \\
(41.4)\end{array}$ & $\begin{array}{c}549.1 \\
(238.9)\end{array}$ & $\begin{array}{l}17.31 \\
(3.25)\end{array}$ & $\begin{array}{c}2.37 \\
(0.11)\end{array}$ & $\begin{array}{c}6.68 \\
(0.91)\end{array}$ & $\begin{array}{l}252.9 \\
(73.0)\end{array}$ & $\begin{array}{c}3.44 \\
(0.28)\end{array}$ & $\begin{array}{c}2.90 \\
(1.04)\end{array}$ \\
\hline
\end{tabular}

${ }^{\mathrm{z}}$ Early yield is that accumulated during the first 6 weeks of harvest.

Table 6. Results for analysis of variance for production and vegetative growth traits of field grown cultivars.

\begin{tabular}{|c|c|c|c|c|c|c|c|c|}
\hline \multirow[b]{2}{*}{ Source } & \multicolumn{8}{|c|}{ Mean squares } \\
\hline & $\begin{array}{l}\text { Early } \\
\text { yield }^{z}\end{array}$ & $\begin{array}{l}\text { Total } \\
\text { yield }\end{array}$ & $\begin{array}{l}\text { Fruit } \\
\text { size }\end{array}$ & $\begin{array}{l}\text { Appearance } \\
\text { score }\end{array}$ & Firmness & $\begin{array}{c}\text { Fall-winter } \\
\text { diam }\end{array}$ & $\begin{array}{l}\text { Spring } \\
\text { relative } \\
\text { growth }\end{array}$ & $\begin{array}{c}\text { Spring } \\
\text { crown } \\
\text { no. }\end{array}$ \\
\hline$\overline{\text { Block }}$ & 2,746 & $143,277^{*}$ & 0.4 & $0.104^{* *}$ & 0.05 & $7,056^{* *}$ & $3.7^{\dagger}$ & $13.8^{* *}$ \\
\hline Vernalization (V) & $8,500^{\dagger}$ & $1,501,542^{*}$ & $81.5^{* *}$ & 0.001 & $0.63^{\dagger}$ & $76,386^{* *}$ & $1.819 .2^{* *}$ & \\
\hline Region (R) & $53,261^{* *}$ & $4,632,847^{* *}$ & $203.7^{* *}$ & $1.687^{* *}$ & 6.42 & $16,268^{*}$ & 14.53 .1 & \\
\hline $\mathrm{V} \times \mathrm{R}$ & 1,381 & 125,587 & 3.7 & $0.055^{*}$ & 0.12 & 2,123 & 2.8 & 0.6 \\
\hline Cultivar (C)/R & $4,748^{\dagger}$ & $428,674^{* * *}$ & 6.8 & $0.201^{* * *}$ & $3.95^{* *}$ & $2,917^{\dagger}$ & $7.1^{* * *}$ & $1.4^{* *}$ \\
\hline $\mathrm{V} \times \mathrm{C} / \mathrm{R}$ & 1,663 & $66,050^{* *}$ & $3.2^{*}$ & 0.013 & 0.31 & $1,121^{* *}$ & 1.10 .3 & \\
\hline Error & 1,336 & 20,392 & 1.4 & 0.013 & 0.29 & 269 & 1.0 & 0.2 \\
\hline
\end{tabular}

${ }^{\mathrm{z}}$ Early yield is that accumulated during the first 6 weeks of harvest.

${ }^{\dagger}, *, * *$ Significant at $P=0.10,0.05$, or 0.01 , respectively.

Table 7. Components of variance and proportions of the phenotypic variance of random effects for vegetative growth and production traits of plants grown in the field.

\begin{tabular}{|c|c|c|c|c|c|c|c|c|}
\hline Source & $\begin{array}{l}\text { Early } \\
\text { yield }^{z}\end{array}$ & $\begin{array}{l}\text { Total } \\
\text { yield }\end{array}$ & $\begin{array}{l}\text { Fruit } \\
\text { size }\end{array}$ & $\begin{array}{l}\text { Appearance } \\
\text { score }\end{array}$ & Firmness & $\begin{array}{l}\text { Fall-winter } \\
\text { diam }\end{array}$ & $\begin{array}{l}\text { Spring } \\
\text { relative } \\
\text { growth }\end{array}$ & $\begin{array}{c}\text { Spring } \\
\text { crown } \\
\text { no. }\end{array}$ \\
\hline \multirow[t]{2}{*}{ Region (R) } & 4,100 & 348,289 & 16.50 & 0.121 & 0.22 & $1,037.8$ & 0.47 & 0.12 \\
\hline & $64 \%$ & $26 \%$ & $32 \%$ & $25 \%$ & $6 \%$ & $12 \%$ & $6 \%$ & $5 \%$ \\
\hline \multirow[t]{2}{*}{ Vernalization $(\mathrm{V}) \times \mathrm{R}$} & 0 & 10,091 & 0.08 & 0.007 & 0 & 169.8 & 0.29 & 0.06 \\
\hline & $0 \%$ & $1 \%$ & $0 \%$ & $1 \%$ & $0 \%$ & $2 \%$ & $4 \%$ & $2 \%$ \\
\hline \multirow[t]{2}{*}{ Cultivar (C)/R } & 771 & 90,656 & 0.90 & 0.047 & 0.91 & 449.0 & 1.50 & 0.28 \\
\hline & $12 \%$ & $7 \%$ & $2 \%$ & $10 \%$ & $26 \%$ & $5 \%$ & $20 \%$ & $11 \%$ \\
\hline \multirow[t]{2}{*}{$\mathrm{V} \times \mathrm{C} / \mathrm{R}$} & 164 & 22,829 & 0.90 & 0 & 0.01 & 426.0 & 0.05 & 0.02 \\
\hline & $3 \%$ & $2 \%$ & $2 \%$ & $0 \%$ & $0 \%$ & $5 \%$ & $1 \%$ & $1 \%$ \\
\hline \multirow[t]{2}{*}{ Error } & 1337 & 860,098 & 33.5 & 0.307 & 2.32 & $6,390.0$ & 5.20 & 2.12 \\
\hline & $21 \%$ & $65 \%$ & $65 \%$ & $64 \%$ & $67 \%$ & $75 \%$ & $69 \%$ & $82 \%$ \\
\hline
\end{tabular}

${ }^{\mathrm{z}}$ Early yield is that accumulated during the first 6 weeks of harvest. 
climates lack the innate capacity to capture the longer growing season of Mediterranean climates.

In addition to greater productivity, our study demonstrated that California cultivars obtained larger vegetative size during the winter and differentiated more crowns than cultivars selected in most other regions. Hancock et al. (1992) found little absolute difference in vegetative attributes, and, when found, these differences suggested larger absolute growth for eastern cultivars. These observations are consistent with California cultivars' possessing a superior capability for winter growth-relative spring growth rates did not differ in our study, but other explanations are possible. Because comparisons of these cultivars in controlled environments detected no temperature $\times$ genotype interaction or temperature $\times$ region interaction effects (Rariden and Shaw, 1993), it is unlikely that the observed growth differences can be explained based on response to growing temperature alone.

All cultivars in our study responded positively in growth and productivity to partial vernalization. However, interactions between regional patterns of growth or performance and vernalization treatment were absent. Our conclusion is that selection in differing environments has had little effect in modifying plant response to chilling. Our test location in Watsonville typically receives 500 to $700 \mathrm{~h}$ at $<7.2 \mathrm{C}$ annually, but this local vernalization would primarily affect the vigor of spring vegetative activity rather than growth during the fall and winter. Because relative spring growth rates did not differ significantly among genotypes from different geographical regions, local chilling seems to be of little consequence.

Germplasm from different regions within North America has been developed to satisfy a range of different priorities. For example, northwestern cultivars have been selected with processing quality as a priority, and the focus of many eastern programs has been resistance to soil diseases (Chandler, 1991). Although infusion of germplasm from other North American regions into California breeding populations is a simple matter in theory, our results suggest that substantial adaptational barriers must be overcome. Non-Californian cultivars are less vigorous and less productive in Mediterranean production environments, and genetic transfer of beneficial characters from these sources may be obtained only after several cycles of recombination and selection.

\section{Literature Cited}

Arney, S.E. 1956. Studies in growth and development in the genus Fragaria: I. Factors affecting the rate of leaf production in Royal Sovereign strawberry. J. Hort. Sci. 28:73-84.

Bringhurst, R.S. and V. Voth. 1984. Breeding octoploid strawberries.
Iowa State J. Res. 58(4):371-381.

Chandler, C.K. 1991. North American strawberry cultivars, p. 60-65. In: A. Dale and J.J. Luby (eds.). The strawberry into the 21 st century. Timber Press, Portland, Ore.

Darrow, G.M. and G.F. Waldo. 1934. Responses of strawberry varieties and species to duration of the daily light period. U.S. Dept. of Agr. Tech. Bul. 453.

Galletta, G.J., A.D. Draper, and J.L. Maas. 1989. Combining disease resistance, plant adaptation, and fruit quality in breeding short day and day-neutral strawberries. Acta Hort. 265:43-51.

Galletta, G.J. and R.S. Bringhurst. 1990. Strawberry management, p. 83156. In: G.J. Galletta and D.G. Himelrick (eds.). Small fruit crop management. Prentice-Hall, Englewood Cliffs, N.J.

Guttridge, G.C. 1953. The influence of some environmental factors on the initiation of flowers and runners in the strawberry plant. $\mathrm{PhD}$ diss. Univ. of Reading, England.

Hancock, J.F. and D.H. Scott. 1989. Strawberry cultivars and worldwide patterns of strawberry production. Fruit Var. J. 42(3):102-108.

Hancock, J.F., S.C. Hokason, P.W. Callow, M. Sakin, K. Haghighi, and J.A. Flore. 1992. Performance of Californian and eastern U.S. strawberry cultivars under conditions mimicking eastern production systems. J. Amer. Soc. Hort. Sci. 117:991-995.

Kronenberg, H.G. and L.M. Wassenaar. 1972. Dormancy and chilling requirement of strawberry varieties for early forcing. Euphytica 21:454-459.

Nicoll, M.F. and G.J. Galletta. 1987. Variation in growth and flowering habits of Junebearing and everbearing strawberries. J. Amer. Soc. Hort. Sci. 112:872-880.

Rariden, J.M., and D.V. Shaw. 1992. Vegetative attributes of North American cultivars with differing temperature regimes. Acta Hort. 348:109-116.

Roberts, M.J., S.P. Long, L.L. Tieszen, and C.L. Beadle 1985. Measurement of plant biomass and net primary production, p. 1-18. In: J. Coombs, D.O. Hall, S.P. Long, and J.M.O. Scurlock (eds). Techniques in bioproductivity and photosynthesis. 2nd ed. Pergamon Press, New York.

Searle, S.R. 1971. Linear models. Wiley, New York.

Shaw, D.V. 1993. Genetic correlations between vegetative growth traits and productivity at different within-season intervals for strawberries (Fragaria $\times$ ananassa). Theoretical Applied Genet. 85:1001-1009.

Sjulin, T.M. and A. Dale. 1987. Genetic diversity of North American strawberry cultivars. J. Amer. Soc. Hort. Sci. 112: 375-385.

Strik, B.C. and J.T.A. Proctor. 1988a. The importance of growth during flower bud differentiation to maximizing yield in strawberry genotypes. Fruit Var. J. 42:45-48.

Strik, B.C. and J.T.A. Proctor. 1988b. Growth analysis of field-grown strawberry genotypes differing in yield: II. The hill system. J. Amer. Soc. Hort. Sci. 113: 899-904.

Voth, V. and R.S. Bringhurst. 1990. Culture and physiological manipulation of California strawberries. HortScience 25:889-892.

Welch, N. 1989. Strawberry production in California. Univ. of California Coop. Ext. Lflt. 2959. 